\title{
When Edwards meets Klinefelter: a new case of double Aneuploidy
}

\begin{abstract}
This article describes a new occurrence of double aneuploidy Edward syndrome/ Klinefelter syndrome in a Lebanese baby boy. Given the fact that Edward syndrome and Klinefelter syndrome are relatively common aneuploidies, to date, scant published cases of the double aneuploidy Trisomy 18/XXY have been described in the literature. The predominant features of Trisomy 18 makes it clinically challenging to diagnose or suspect Klinefelter syndrome when these two conditions coexist. Our infant had features of trisomy 18 with overlapping features of Klinefelter. The prognosis is poor, the genetic counseling remains challenging and the possibility of combined autosome and sex chromosome aberrations should be raised even in the absence of clinical manifestations of the latter.
\end{abstract}

Volume 9 Issue 2 - 2019

\author{
Ziad Chebel, Stephanie Krayem, Yolla Nassif, \\ Dany Al Hamod \\ Department of Pediatrics, Saint George Hospital University \\ Medical Center, University of Balamand, Lebanon
}

Correspondence: Dany Al Hamod, Department of Pediatrics, Saint George University Hospital, University of Balamand, Beirut, Lebanon P.O. Box: 166378; Tel +961-368-3209, Email dany_alhamod@hotmail.com

Received: March 19,2019 | Published: April II, 2018

\section{Introduction}

The presence of abnormal numbers of a chromosome, known as Aneuploidy, is commonly encountered in the clinical practice of Pediatrics. Although Down syndrome (Trisomy 21) is still the most common example of aneuploidy, rarer forms include Patau syndrome (Trisomy 13), Edward syndrome (Trisomy 18), Turner syndrome $(45 \mathrm{X} 0)$ and Klinefelter syndrome (47XXY). Double aneuploidy, is a rare chromosomal abnormality detected after birth since most reported cases are presented in the form of one lethal aneuploidy and end in early miscarriage. ${ }^{1-3}$ It is extremely rare to find living newborn cases with double autosomal trisomy or autosomal trisomy with sex chromosome monosomy or trisomy. ${ }^{4,5}$ Knowing that Edward syndrome and Klinefelter syndrome are relatively common aneuploidies, to date, scant published cases of the double aneuploidy Trisomy 18/XXY have been described in the literature. ${ }^{6}$ This case report describes a new occurrence of double aneuploidy Edward syndrome/Klinefelter syndrome in a Lebanese baby boy. The present case is the first reported case of double aneuploidy in Lebanon.

\section{Case report}

A day 8 of life baby boy was referred to our university hospital for management of respiratory distress. He was born at 39 weeks gestational age by normal vaginal delivery to non-consanguineous, healthy, Gravida 6 Aborta 1 Para 5, 35 year old mother and 38 year old father. Spontaneous abortion occurred during the first pregnancy at 12 weeks of gestation for unknown reasons. The living children are all healthy. The pregnancy was uneventful except for left hydronephrosis and intrauterine growth restriction diagnosed prenatally. Baby was born with an Apgar score 2 and 4 at 1minute and 5minutes respectively, requiring resuscitation and placed on mechanical ventilation. Birth weight was 1800 grams $(<3$ rd percentile $)$, birth length $40 \mathrm{~cm}(<3 \mathrm{rd}$ percentile), head circumference $29 \mathrm{~cm} \quad(<3 \mathrm{rd}$ percentile). Baby remained intubated for 8 days in the peripheral hospital, with failure of weaning from mechanical ventilation which was attributed to a cardiac etiology. Patient was then transferred to our university hospital for further workup and management. Physical examination revealed typical characteristics of trisomy 18: strawberry shaped head, protruding occiput, depressed nasal bridge, high-arched palate, microretrognathism, low set ears (Figure 1A), short neck, wide-spaced nipples, clinodactyly of the 5th fingers, clenched fist (Figure 1B), rocker-bottom feet (Figure 1C) and grade IV pansystolic murmur with thrill. He had descended testes bilaterally with normal size phallus. Neurological exam revealed a hypotonic baby with normal reflexes. Echocardiography revealed the presence of VSD $(6 \mathrm{~mm})$ with left to right shunt, with a PDA of $2.5 \mathrm{~mm}$, dilated left atrium and pulmonary hypertension of $45 \mathrm{mmHg}$. Ultrasound of the brain showed no abnormalities. Ultrasound of the kidneys showed left kidney hydronephrosis with a renal pelvis of $8.7 \mathrm{~mm}$ and dilation of the calyceal system. There was also a left megaureter measuring 12 $\mathrm{mm}$ in diameter. The right kidney was well differentiated and normal in size. Complete blood count, electrolytes, metabolic screening and blood glucose were all normal. Chromosomal analysis of a peripheral blood sample using Giemsa trypsin (GTG banding) showed the karyotype of 48, XXY, +18 (Figure 2). Genetic counselling was offered to the parents. Baby is currently 1 month old, still on noninvasive mechanical ventilation and feeding by nasogastric tube with failure to gain weight.

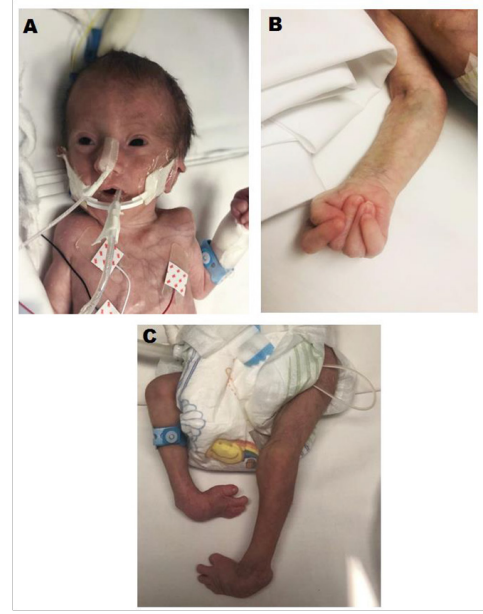

Figure I Patient at day 26 of life: (A) Strawberry shaped head, protruding occiput, high-arched palate, microretrognathism, low set dysplastic ears; (B) Clenched hands with clinodactyly of the 5 th finger; (C) Rocker bottom feet. 


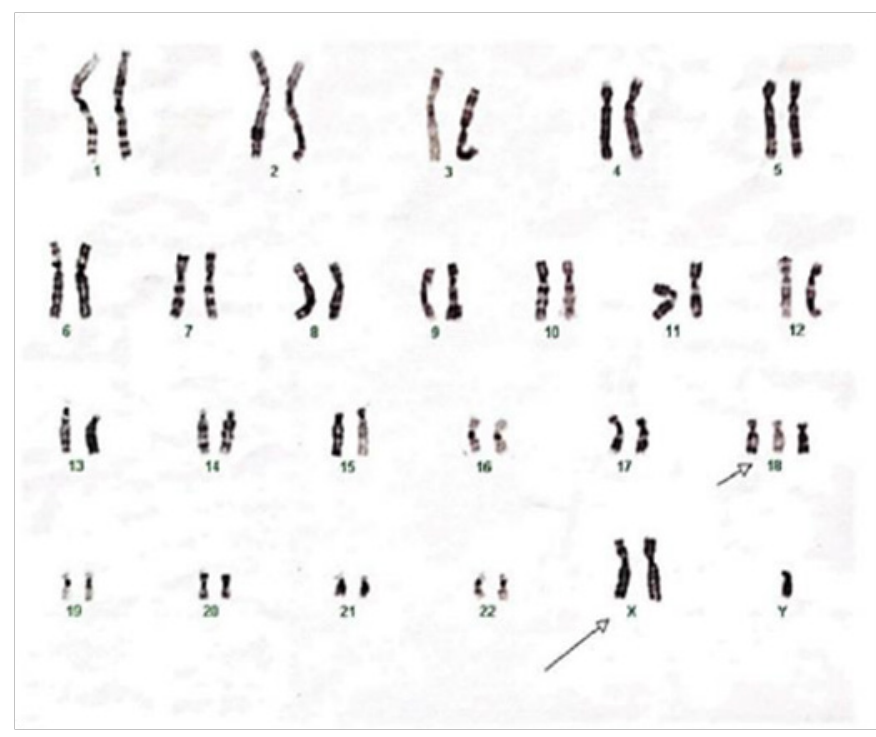

Figure 2 The chromosome analysis showing the karyotype of $48, X X Y,+18$.

\section{Discussion}

Few cases were found in the literature reporting the coexistence of Edward and Klinefelter syndromes. To our knowledge this rare double aneuploidy is the first reported case in Lebanon. With the predominant features of Trisomy 18, it is clinically very challenging to diagnose or suspect Klinefelter syndrome when these two conditions coexist. In fact, in sex chromosomal abnormalities, the clinical manifestations are usually missing or appear later in life after puberty. ${ }^{7}$ The parental origin of extra chromosomes and cell division level where non disjunction occurred were not assessed in our case. However, there is a clear association in literature between double aneuploidies and advanced maternal age. The extra chromosomes are mostly from maternal origin and the non-disjunction events can occur in different cell division stages. ${ }^{6-8}$ There is a clear association between the advanced maternal age and chromosome laxity leading to no disjunction. This is mainly due to inappropriate chromosome coiling and condensation in the oocyte of older women. ${ }^{9}$ In our case, our baby had features of trisomy 18 with overlapping features of Klinefelter. The typical facial features included strawberry shaped head with prominent occiput, microretrognathism, dysplastic low set ears. He also had a short neck, wide-spaced nipples, clinodactyly, clenched fist and rockerbottom feet which are characteristic findings in trisomy 18. Patient also had long limbs which is characteristic in Klinefelter and seen in most reported cases Trisomy 18/XXY. It is common to have cardiac abnormalities in Trisomy 18 but less so on Klinefelter, however in the association of both trisomies, all the reported cases had cardiac involvement typically seen in Trisomy 18 (PDA and VSD in more than $95 \%$ of cases). ${ }^{10}$ Our patient had a grade IV pansystolic murmur with thrill and found to have a VSD with left to right shunt, with a PDA, dilated left atrium and pulmonary hypertension. Although it is common to see cryptorchidism in both Trisomy 18 and Klinefelter, our patient had descended testes bilaterally with normal size phallus. Renal involvement typically seen in Trisomy 18 but absent in Klinefelter was also noted in our case with left kidney hydronephrosis and dilation of the calyceal system. While the prenatal diagnosis remains difficult, findings of polyhydramnios, IUGR, increased fetal nuchal translucency and major cardiac structural abnormality on prenatal ultrasound should raise concerns about the possibility of a chromosomal abnormality. ${ }^{10}$ With only one case of the double aneuploidy Trisomy 18/XXY reported as living beyond 15 months of age, ${ }^{11}$ the prognosis is poor and the genetic counselling remains challenging. ${ }^{12-14}$ In conclusion, while the coexistence of the double aneuploidy Trisomy $18 / \mathrm{XXY}$ is rare, the possibility of combined autosome and sex chromosome aberrations should be raised even in the absence of clinical manifestations of the latter.

\section{Acknowledgments}

None.

\section{Conflicts of interest}

The authors declared there is no conflicts of interest.

\section{References}

1. Jones KL. Trisomy 18 syndrome. Smith's Recognizable Patterns of Human Malformation. 6th ed. Philadelphia: Elsevier Saunders Company; 2006. $13-17 \mathrm{p}$.

2. Nielsen J, Vetner M, Holm V, et al. Klinefelter's syndrome and trisomy 18 in a newborn boy. Clin Genet. 1978;13(3):259-64.

3. MacFaul R, Turner T, Mason MK. Down's/Turner's mosaicism. Double aneuploidy as a rare cause of missed prenatal diagnosis of chromosomal abnormality. Archives of Disease in Childhood. 1981;56(12):962-963.

4. Haylock JM, Fitzgerald DD, Dennett X. Two cases of the 18-trisomy syndrome, one in combination with an XXY karyotype. Hum Chromos Newsl. 1963;1(9):15-16.

5. Zellweger H, Abbo G. Double trisomy and double trisomic mosaicism. Am J Dis Child. 1967;113(3):329-337.

6. Chen CP, Chern SR, Chen CY, et al. Double aneuploidy with Edward-Klinefelter syndrome $48, \mathrm{XXY},+18$ ) of maternal origin:prenatal diagnosis and molecular cytogenetic characterization in a fetus with arthrogryposis of the left wrist and aplasia of left thumb. Taiwan J Obstet Gynecol. 2011;50(4):479-484.

7. Weeraesekara KP, Anjana Sirisena. Coexisting Edward syndrome and Klinefelter syndrome. Sri Lanka J Child Helth. 2013;42(3):170-172.

8. Diego-Alvarez D, Ramos-Corrales C, Garcia-Hoyos M, et al. Double trisomy in spontaneous miscarriages: cytogenetic and molecular approach. Hum Reprod. 2006;21(4):958-966.

9. Hulten MA. The origin of aneuploidy: Bivalent instability and the maternal age effect in trisomy 21 Down syndrome. Am J Med Genet Suppl. 1990;(suppl 7):160-161.

10. De Vigan C, Baena N, Cariati E, et al. EUROSCAN Working Group. Contribution of ultrasonographic examination to the prenatal detection of chromosomal abnormalities in 19 centres cross Europe. Ann Genet. 2001;44(4):207-217.

11. Hou JW. Double aneuploidy: trisomy 18 and XXY in a boy. Chang Gung Med J. 2006;29(4):6-12.

12. Cohen MM, Bumbalo TS. Double aneuploidy: trisomy-18 and Klinefelter's syndrome. Am J Dis Child. 1967;113:483-486.

13. Henchman DC, Grey J, Campbell JB, et al. Klinefelter's syndrome with mosaicism trisomy-18. Aust Paediatr J. 1970;6(3):142-145.

14. Bach C, Toublanc JE, Gautier M. Case of double aneuploidy:trisomy 18 and XXY. Ann Genet 1973;16(1):61-66. 